\title{
Kernos
}

Revue internationale et pluridisciplinaire de religion grecque antique

$11 \mid 1998$

Varia

\section{Franz Cumont, un fondateur}

\section{Robert Turcan}

Édition électronique
URL : http://journals.openedition.org/kernos/1230

DOI : 10.4000/kernos. 1230

ISSN : 2034-7871

\section{Éditeur}

Centre international d'étude de la religion grecque antique

Édition imprimée

Date de publication : 1 janvier 1998

ISSN : 0776-3824

\section{Référence électronique}

Robert Turcan, «Franz Cumont, un fondateur », Kernos [En ligne], 11 | 1998, mis en ligne le 21 avril 2011, consulté le 22 avril 2019. URL : http://journals.openedition.org/kernos/1230 ; DOI : 10.4000/ kernos. 1230 
Kemos, 11 (1998), p. 235-244.

\section{Franz Cumont, un fondateur*}

Commémorer la mort de F. Cumont, c'est d'abord célébrer une œuvre qui n'est pas morte. Car le cinquantenaire de cette mort coïncide (à un an près) avec le centenaire d'un livre majeur, qui allait vivifier et faire fructifier comme un nouveau rameau d'or sur l'arbre de l'histoire: le tome II des Textes et monuments figurés relatifs aux mystères de Mithra.

Il s'agissait d'un corpus, et ce corpus ne concernait, après tout, qu'un dieu parmi d'autres : pire ! un de ces dieux immigrés, déracinés ou, du moins, étrangers à l'antiquité classique et dont la tradition littéraire nous parle si peu au total... Il est vrai que certains Pères de l'Église s'y intéressent à leur manière, et c'est évidemment l'indice d'une vitalité singulière : on ne s'en prendrait pas avec une pareille ardeur à un culte purement marginal ou étroitement confidentiel.

Fait curieux, mais significatif : le tome II paraissait en 1896, trois ans avant le tome I, dont la publication parachèverait le faîte de l'édifice en le dotant d'un fronton lumineux, explicatif, et qui devait longtemps servir de « matrice » à notre vision du mithriacisme. Au vrai, sous le titre d'Introduction, ce tome premier consistait, après la Critique des documents inventoriés, à présenter des Conclusions. Pourtant (et non sans quelque contradiction d'apparence), F. Cumont s'en expliquait au lecteur : "Ce livre n'est pas une conclusion, mais un prologue "; façon de nous dire qu'il serait comme un marchepied désormais nécessaire à quiconque voudrait tenter d'avoir un certain nombre d'idées claires sur un dossier qu'embarrassaient déjà quantité d'hypothèses aussi dissemblables que déroutantes. L'auteur se tiendrait pour satisfait, précisait-il, s'il avait "réussi à montrer l'inanité des interprétations vagues ou fausses que l'on allait répétant et, en dégageant le terrain des broussailles qui l'encombraient, à ouvrir la voie aux investigations futures ". Ces Conclusions allaient presque immédiatement être rééditées, allégées des notes trop savantes, sous le titre : Les mystères de Mitbra.

Revenons cependant plus précisément au centenaire du tome II des Textes et monuments. Asssurément, nous savons tous que rien ne vieillit plus vite qu'un corpus, presque toujours périmé du jour même où il est

* Cet article reproduit le texte dune conférence présentée aux Facultés Notre-Dame de la Paix à Namur le 17 mars 1997, lors de l'assemblée générale annuelle de la Société belgo-luxembourgeoise d'Histoire des Religions. 
publié ! F. Cumont n'avait pas plus tôt achevé le sien qu'il avait dû l'assortir d'un Supplément, non pas seulement en raison de découvertes récentes (comme celles des Mitbraea de Carnuntum en 1894 et de Sarrebourg en 1895 ou de tels reliefs connus l'année même de la parution des Textes et monuments), mais afin de compléter scrupuleusement certaines données de la tradition littéraire, épigraphique ou figurée, sinon pour retoucher ou rectifier ses notices. D'emblée, F. Cumont reconnaissait que son recueil de matériaux était déjà incomplet à l'heure où il écrivait sa Préface : "Ce sera néanmoins (déclarait-il) la partie la plus durable de mon travail, parce qu'elle est la plus impersonnelle ».

Affirmation a priori paradoxale, où l'on sent la discrétion, la pudeur du savant qui en 1947, quelques mois avant sa mort, fut surpris et comme " interloqué », lorsqu'on lui demanda " comment il se représentait son œuvre et la place qu'elle tiendrait dans l'histoire des religions ». Il paraît qu'alors le visage de F. Cumont se détendit, " comme en signe d'acquiescement, et il prit l'air modeste et recueilli d'une chatte à qui l'on fait compliment de sa nichée... » Mais, modestie mise à part, il n'avait sans doute pas tort en un sens de penser qu'en colligeant les matériaux du tome II, il avait fait œuvre durable. Car le principe même du corpus était alors exemplaire et novateur. Dans une recherche, rien n'est essentiel comme l'inventaire contrôlé, raisonné, comparé, critique et méthodique de toute la documentation matérielle, écrite, inscrite ou figurée.

Il y avait, certes, des précédents depuis que l'Italien G. Symeoni avait donné, en 1558, la première reproduction gravée d'un monument mithriaque : un relief trouvé à Lyon, que F. Cumont disait « sans importance », à tort (me semble-t-il), car il aurait pu mettre le savant belge sur la voie d'une réflexion en contradiction - il est vrai - avec certaines de ses interprétations aujourd'hui contestées (ce qui donnerait raison à la phrase précitée de sa Préface sur la part moins « impersonnelle » et donc plus discutable de son œuvre). En 1700, Th. Hyde dans sa Veterum Persarum et Partborum ei Medorum religionis bistoria avait commenté plusieurs témoignages de la tradition littéraire gréco-romaine et reproduit quatre seulement des bas-reliefs connus au siècle précédent. Un peu plus tard, l'Antiquité expliquée de B. de Montfaucon en donnerait davantage, mais sans rigueur critique dans le choix des monuments.

Le premier, G. Zoëga dans ses Abbandlungen publiées en 1817 par F.G Welcker a répertorié en les expliquant cinquante-deux images de Mithrí Tauroctone et dix autres du Léontocéphale, non sans une relative sagacite dont F. Cumont retiendra la marque. Mais les planches in-folio données er 1847 par F. Lajard dans son Introduction à l'étude du culte public et de. mystères de Mitbra allaient demeurer durant un demi-siècle l'unique recuei ou, pour reprendre les mots mêmes de F. Cumont, "le fondement de toute les recherches sur le mithriacisme » (seul E. Majonica regroupera systémati quement après lui, en 1878, les représentations de Mithra pétrogène) Pourtant, cet «Atlas » de F. Lajard - incomplet et souvent tributaire de publi 
cations antérieures «plus ou moins infidèles » (F. Cumont) - ne consignait qu'une cinquantaine de statues ou reliefs et seulement deux plans de Mitbraea! Surtout, F. Lajard, influencé par la Symbolique de F. Creuzer et enclin à se fourvoyer dans un comparatisme arbitraire, n'avait ni « sens critique » ni « esprit historique », pour citer encore F. Cumont. Quant aux textes grecs et latins, F. Windischmann et T. Fabri les avaient bien déjà colligés, le premier en 1857, le second en 1883. Mais ces travaux (notamment la dissertation de T. Fabri) appelaient une révision attentive et argumentée.

Faire un vrai corpus exige un ordre, une perspective, une démarche heuristique et herméneutique, une architecture qui ne sont pas à la portée de tous les chercheurs, même courageux et honnêtement intentionnés. Suivant le mot de Montaigne qu'E. Rohde citait en réponse au pamphlet de Wilamowitz contre Nietzsche, "il est impossible de renger les pieces à qui n'a une forme du total en sa teste " (Essais, II, 1). N'importe qui peut accumuler des informations: de nos jours, c'est l'ordinateur qui enregistre et risque de donner aux ignares l'illusion d'une mémoire. Mais, comme le soulignait la recension que J. Réville fit en 1901 des Textes et monuments dans la Revue de l'Histoire des Religions, on ne saurait " confondre un fouillis de documents avec de l'histoire... Le vrai talent, c'est de savoir les employer chacun à sa place, sans les déformer, mais aussi en ne leur accordant que la place qui leur revient ». En ce sens, l'Introduction du tome I précédait bien (en l'inspirant) le tome II, même si ce tome II paraissait trois ans plus tôt, en tant que socle étayant la grande façade des Conclusions.

F. Cumont y marquait, en effet, le souci d'une documentation exhaustive et intégrale. Dès 1892 et 1893, dans deux livraisons de la Reuue Archéologique, il avait donné un Catalogue sommaire des monuments figurés relatifs au culte de Mithra, avec plus de trois cents numéros, y compris les pierres gravées et les bijoux. Mais en 1896, non seulement le nombre des représentations s'augmentait d'une centaine (ce qui faisait désormais environ quatre cents monuments figurés), mais aux deux . Hitbraea de F. Lajard s'en ajoutaient dix-sept, et $\mathrm{F}$. Cumont rassemblait quelque six cents inscriptions. De plus, le corpus des données matérielles était précédé d'un recueil des textes orientaux, grecs ou latins, ces derniers avec un apparat critique (mais sans traduction). Il faut observer, enfin, que beaucoup dillustrations photographiques permettaient de vérifier le détail des monuments, au lieu de faire confiance à l'œil du dessinateur. même si la qualité des reproductions demeurait encore inégale. Sur cette base pouvait s'ouvrir, selon J. Réville, « un chapitre nouveau de l'histoire des religions".

F. Cumont avait le sentiment que son édifice des Conclusions (qu'il considérait comme un "prologue ") aurait moins de longérité que les fondations appareillées dans ce tome II aujourdhui centenaire. Et, de fait. beaucoup de ses interprétations ont été remises en cause depuis une quarantaine d'années. Dans un pareil livre d'images dont le texte était perdu, mais en partie restitué conjecturalement par les Conclusions. un certain nombre de points pouvaient susciter des interrogations sur le bien- fondé des exégèses 
qu'y développait le savant belge. Mais la conception même de l'inventaire occupant le tome II (et qui témoigne d'une science prodigieuse chez cet homme qui n'avait pas encore atteint la trentaine) n'était pas à l'abri de tout reproche dans la mesure où le corpus préjugeait de plusieurs « conclusions » inégalement convaincantes.

Pour s'en tenir à la lettre du titre Textes et monuments figurés relatifs aux mystères de Mitbra, on ne devrait $\mathrm{y}$ trouver que la documentation concernant strictement cette religion à «mystères» qui n'émerge dans le monde romain qu'à dater du rer siècle avant J.-C. (si l'on en croit Plutarque), mais seulement vers la fin du siècle suivant, si l'on tient compte de deux vers de Stace et de l'épigraphie proprement mithriaque. Le phénomène et la multiplication des rituels initiatiques appartiennent au temps, au "milieu » de la civilisation hellénistique, avec des prolongements et des modalités diverses qui ne s'affirment expressément qu'à l'époque romaine impériale. Le gros problème - qui n'est toujours pas résolu - reste de savoir où, quand, comment s'est élaboré un culte mystérique patronné par Mithra.

Or c'est en fonction d'hypothèses très personnelles que $F$. Cumont intègre à son corpus maints textes orientaux, grecs ou latins, sans rapport évident et direct avec les "mystères » à proprement parler. Nous référant aux écrits arméniens, il nous dit que "placés sur la route qui a conduit Mithra du plateau de l'Iran à la Méditerranée, ils nous apprendront, du moins on peut l'espérer, par quelles formes successives son culte a passé avant d'aboutir aux mystères occidentaux ». Mais y eut-il des mystères « orientaux »? ou ces « mystères occidentaux » procèdent-ils vraiment d'une tradition orientale ? On en débat, et non sans raison. « Le nom grec de 'mystères', nous dit F. Cumont, ne doit point faire illusion... . En Perse même, les mages formaient une caste fermée, qui paraît avoir été divisée en plusieurs classes subordonnées », et de supposer qu'en terre étrangère ces « mages hellénisés » auraient d'abord conservé jalousement leurs « dogmes cachés», avant de les communiquer moyennant des cérémonies secrètes à quelques initiés. D'où la prise en compte des testimonia relatifs aux mages d'Asie Mineure, textes où il n'est pas question de «mystères » et où le nom même de Mithra n'est pas énoncé. Mais tous les textes où son nom apparaît n'ont pas pour autant un rapport nécessaire avec les «mystères" mêmes de Mithra. On évoque ou invoque le Soleil sous ce nom d'allure exotique sans songer toujours précisément au Tauroctone.

En revanche, F. Cumont n'a pas reproduit les passages de l'Avesta où Mithra est invoqué, en particulier le Mihr-Yasht, ni les textes pehlevis, persans et arabes où (il le reconnaît lui-même) "s'est conservé parfois le souvenir de traditions fort anciennes. Un pareil travail nous eût entraîné fort loin... » Aujourd'hui, on y joindrait sans doute la littérature sogdienne, sinon les textes manichéens de Turfan, puisquaussi bien F. Cumont tenait à reconnaître dans le manichéisme un héritier du mithriacisme. Mais un corpus de cette ampleur ne pourrait être utilement critique qu'à condition de mobiliser l'acquisition de compétences excédant la vie d'un homme, même s'il se fait 
moine ! Et l'on est en droit de se demander si ce corpus nous aiderait à mieux discerner la genèse et la prime jeunesse du Mithra gréco-romain.

Quant à l'épigraphie ou l'iconographie, le catalogue du tome II outrepassait chronologiquement aussi bien que géographiquement l'aire des mystères mithriaques, puisqu'il annexait les inscriptions achéménides de Suse et de Persépolis, les documents de Commagène ou les monnaies koushanes : à ce compte, il faudrait faire état aujourd'hui de la sculpture et de la sigillographie sassanides ou des images sogdiennes d'un dieu solaire en char qu'on identifie avec Mithra...

Quoi qu'il en soit, une somme aussi fortement mûrie et pensée, sa mise en œuvre, sa cohérence intellectuelle et sa totalité aussi harmonieuse que lumineuse avaient de quoi séduire plusieurs générations d'antiquisants, qu'ils fussent archéologues, philologues ou historiens. Personne n'a fait ou refait un travail comparable à celui de $\mathrm{F}$. Cumont. Les critiques auxquelles il s'exposait restent à la mesure de l'ampleur même de son propos et d'une science inégalée. Il n'aurait pas prêté le flanc à des remises en cause s'il n'avait pas embrassé son sujet avec une généreuse intelligence. Il n'est pas, je crois, de grand historien qui ne doive passer un jour ou l'autre pour un visionnaire.

C'est le tome I des Textes et monuments qui nous a tous marqués et continue de nous marquer. En vérité pourtant, F. Cumont avait raison de nous donner son corpus comme « la partie la plus durable » de son opus magnum.

Ce fut d'abord un modèle « édifiant » au sens propre et figuré du terme, non pas seulement pour l'étude du mithriacisme, mais de tous les cultes du monde romain. La thèse de G. Lafaye sur les Divinités d'Alexandrie bors d'Égypte (1884) avait certes esquissé l'exemple d'un « catalogue » annexé à la synthèse, mais sans l'exigence systématique des dénombrements « cartésiens ». Les inventaires qu'on trouvait dans le Lexikon de W.H. Roscher laissaient encore à désirer, quoique déjà fort précieux. Cependant, la science allemande manifestait depuis plusieurs décennies le souci de rassembler et d'ordonner les matériaux: Monumenta Germaniae Historica, CIL et CIG, corpus des sarcophages romains, attiques ou micrasiatiques (dont le tome II avait paru en 1890). C'est à cette école allemande que s'était mis F. Cumont, après avoir pris ses grades de docteur en Philosophie et Lettres à l'Université de Gand en 1887 (il n'avait pas vingt ans). Nous savons qu'il suivit les enseignements de Th. Mommsen et de H. Usener, peut-être aussi de Wilamowitz, mais qu'il connut également $E$. Rohde, alors à Heidelberg où il achevait de méditer son admirable Psycbe. À Paris même, il fréquenta l'École des Hautes Études où, depuis 1886; fonctionnait une section des « Sciences Religieuses ".

Ce pluralisme d'influences, de méthode et de formation nous explique, en somme, que F. Cumont ait su concilier la rigueur des fichages et classifications documentaires (à l'allemande) avec une perspective généreusement compréhensive envers les phénomènes d'ordre religieux ou spirituel. G. Showerman - le même qui révisera les derniers chapitres d'After Life in 
Roman Paganism en 1923 - reconnaissait dans les Textes et monuments la conjonction de la patience allemande avec l'intuition latine, «the characteristics of both German and French scholarship ». Au vrai, F. Cumont excellait dans l'art de faire dialoguer les voix des différentes traditions (littéraire, épigraphique, archéologique) pour les accorder dans un chœur harmonieux et puissant. «L'archéologie, si elle est privée du secours de la philologie, devient une science conjecturale » exposée aux « interprétations arbitraires », rappelait-il quelques mois avant sa mort. On sait la définition que S. Reinach donnait de l'archéologie : "explication des monuments par les textes et des textes par les monuments ». Elle aurait pu servir de devise à F. Cumont ou d'épigraphe à ses œuvres maîtresses jusqu'à Lux perpetua. Toute histoire, y compris surtout celle des idées ou des mentalités, requiert l'examen des relations diverses et complexes qui se tissent dans la vie et l'environnement des hommes en société. Il n'y a donc d'histoire que totale. Toutefois, il importe aussi de prendre garde au fait que telle source d'information peut correspondre à une tradition singulière. C'est ainsi qu'on est en droit de constater certaines divergences entre le témoignage indirect de Porphyre sur les mystères mithriaques et le témoignage direct du matériel épigraphique ou archéologique. Il faut comparer pour distinguer.

Depuis un siècle, le corpus de F. Cumont en a inspiré beaucoup d'autres, et dès 1909 l'ouvrage d'A. H. Kan sur Jupiter Dolichenus - que compléterait en 1951 le Répertoire de P. Merlat dont la thèse est dédiée « À la mémoire de F. Cumont ». Depuis, les Cavaliers Danubiens, Mên, Apis, Zeus Héliopolitain, le Cavalier Thrace, Sabazios, Cybèle et Attis ont fait l'objet d'utiles recueils dans la Collection des EPRO qui, grâce à M. J. Vermaseren, a pour ainsi dire multiplié la postérité de F. Cumont. Nombreux surtout ont paru les inventaires locaux ou régionaux. Mais depuis 1896, l'archéologie avait enrichi considérablement le dossier mithriaque. F. Cumont lui-même avait fait connaître ou commenté plusieurs découvertes. C'est donc au Maître Francisco Cumont qui vita sua exemplum dedit - que M.J. Vermaseren a dédié en 1956 son «aggiornamento »: il n'y réactualisait que le répertoire épigraphique et archéologique, mais sans isoler - comme F. Cumont avait eu tort de le faire - les inscriptions des monuments.

Tel que F. Cumont l'a conçu en fonction de ses Conclusions, le tome II des Textes et monuments était donc fécond à bref ou à long terme.

En premier lieu, il permettait de mieux appréhender le phénomène mithriaque dans sa dimension géographique, avec ses zones de densité relative à Rome même ou à Ostie, sur le limes rhénan et danubien. Une carte détaillée ponctuait les indices de cette propagation. À cet égard, les cartes que M. J. Vermaseren nous donnerait soixante ans plus tard des Mitbriaca dans chaque province de l'Empire ne pourraient qu'améliorer notre vision des faits.

En deuxième lieu, outre la diffusion spatiale du mithriacisme, sa pénétration dans les différentes couches de la société romaine apparaissait dès lors 
beaucoup plus clairement. En imposant un réexamen de tous les cas particuliers de dévotion, le corpus attirait l'attention sur les croyants tout autant que sur les croyances, leur condition juridique et (ou) économique, leur appartenance à la hiérarchie militaire ou administrative, leur place à la cour impériale, dans la vie municipale, commerciale ou artisanale, leur mobilité, leur rayon d'action ou d'influence. On revenait sur terre après avoir erré, vagabondé, glosé sur une théosophie héritée de F. Creuzer. G. Boissier n'avait pas ignoré ces problèmes dans sa Religion romaine d'Auguste aux Antonins (1874), mais il n'en avait pas traité sur la base d'inventaires systématiques. Des ouvrages récents, comme les Cultores Mitbrae (1992) de M. Clauss, sont en germe dans le livre même qu'ils critiquent ou rectifient.

En ce qui regarde l'épigraphie, F. Cumont en avait justement souligné tout ensemble l'intérêt et les limites. On connaît sa controverse avec J. Toutain. Les inscriptions ne nous renseignent guère sur le contenu et le sens des actes cultuels, du moins celles qu'on pouvait alors collationner, car les vers peints dans le Mithraeum de S. Prisca nous ont livré sur ce point des révélations significatives.

Toutefois, si le corpus épigraphique de F. Cumont autorisait à mettre l'accent sur la situation des croyants dans l'Orbis Romanus et le réseau des relations humaines, c'est l'analyse comparée des monuments figurés qui devait nous élucider quelque chose des croyances. Il n'y a pas de religion positive sans représentations, sans un imaginaire qui porte mentalement et fixe ou magnifie un «credo ». Ces statues, ces panneaux peints ou sculptés qu'élucident assez rarement des légendes servaient à un enseignement par l'image pour des fidèles qui ne savaient pas toujours lire et écrire. Les reliefs bifaces pivotaient pour correspondre iconographiquement aux différentes phases du service liturgique.

Dans ses Mystères de Mithra, F. Cumont a consacré à l'art des antres un substantiel appendice correspondant au remaniement des p. 213 à 220 du premier tome de ses Textes et monuments, mais à une époque où l'on ne savait rien des peintures mithriaques de Capoue, du Palazzo Barberini, de $S$. Prisca... Il ne prétendait pas avoir acquis « un déchiffrement rigoureusement exact de ces hiéroglyphes ", écrivait-il en 1899. Mais il s'interrogeait opportunément sur la finalité de cette iconographie. "Les symboles disgracieux ou indifférents, dont nos monuments attestent l'emploi multiplié, ne séduisaient point par leur élégance ou leur noblesse, ils fascinaient l'esprit par l'attrait troublant de l'inconnu, et provoquaient dans les âmes la crainte respectueuse d'un mystère auguste ». Plus tard, dans ses Religions orientales, F. Cumont, rappelant que l'archéologie « doit s'efforcer de combler les lacunes énormes que laisse la tradition écrite », se dira convaincu que «l'art religieux de la fin du paganisme... exprime par des symboles les conceptions subtiles de la théologie ». Les progrès de l'illustration photographique, dont le tome II des Textes et monuments faisait bénéficier le lecteur, contribuaient dès lors à le mieux instruire des mythes ou des dogmes impliqués par l'iconographie. Le détail même du style pouvait l'aider à entrer dans la ferveur des fidèles. La 
Préface des Religions orientales en leur $4^{\mathrm{e}}$ édition (1929) y insistait pour finir : «Cette illustration n'est pas une simple parure. Les œuvres de l'art frappent plus notre imagination, nous mettent en contact plus direct avec le passé que celles de la pensée traduite par l'écriture ».

En consignant, enfin, tous les indices matériels que fournissent les sites mithriaques, qu'ils aient concerné l'aménagement interne des cryptes avec leurs annexes, les résidus alimentaires ou le détail du mobilier cultuel, F. Cumont se donnait les moyens de reconstituer au moins hypothétiquement certains points du rituel, qu'on avait eu tendance à négliger ou ignorer. L'exemple des Textes et monuments demeure, à cet égard, d'autant plus incitatif qu'on ne paraît pas avoir épuisé le traitement de cette information.

Dès lors, aussi bien l'épigraphie que la topographie et l'iconographie autorisaient à mieux cerner les rapports de Mithra avec d'autres dieux, le contexte politico-religieux du mithriacisme, la cohabitation ou l'opposition des cultes. S'il n'y a pas d'explication historique d'un phénomène en dehors d'une totalité à la fois diachronique et synchronique, c'est vrai aussi et surtout des variantes du paganisme gréco-romain. On n'y saurait abstraire une divinité pour l'étudier isolément, sans tenir compte des autres dévotions, souvent corrélatives. F. Cumont était enclin, semble-t-il, à surinterpréter certaines constatations de fait. Ainsi, la solidarité de Cybèle et du Tauroctone n'est pas toujours aussi évidente qu'il nous l'affirme, et l'idée que le service de la Grande Mère aurait compensé pour les femmes leur exclusion des antres n'emporte guère finalement la conviction, non plus d'ailleurs que l'emprunt supposé du taurobole au culte d'Anaïtis. L'hypothèse d'une sourde animosité entre isiaques et mithriastes n'apparaît pas mieux fondée.

En revanche, le corpus confirmait les liens du culte persique avec le culte impérial. Il est certain que les valeurs du mithriacisme s'intégraient sans peine dans l'idéologie païenne qui animait le loyalisme envers le pouvoir souverain. On peut seulement douter que le bvarenô avestique ait joué dans ce contexte le rôle que veut lui reconnaître $\mathrm{F}$. Cumont. On a du mal à le suivre en particulier lorsqu'il écrit : «Il y eut un moment où le césarisme parut sur le point de se transformer en un khalifat ». Il songe à Dioclétien, dont le nom apparaît dans la phrase suivante à propos de sa cour qui aurait offert des ressemblances avec celle de Chosroès ... O. Spengler, qui avait lu $\mathrm{F}$. Cumont, écrit aussi - moins de vingt ans après (en 1917) - que Dioclétien est « le premier des khalifes ». Aujourd'hui une pareille affirmation nous surprend. Mais ici surgit un problème inhérent à l'intuition majeure de F. Cumont. Or c'est, je crois, la lecture d'E. Renan qui la lui a suggérée.

F. Cumont se défend d'avoir eu « la prétention d'offrir un tableau de la chute du paganisme ». Mais il avoue immédiatement que, dans les phases diverses de la lutte entre l'idolâtrie et l'Église grandissante, il retient « un épisode de cette révolution décisive », ce qui le conduit à poser la question : «Comment et pourquoi une secte du mazdéisme faillit sous les Césars devenir la religion prédominante de l'empire?» 
On ne pouvait répondre à cette interrogation sans situer le mithriacisme et en apprécier l'impact par rapport aux autres cultes, y compris le christianisme. "Il sembla un instant que le monde dût devenir mithriaste », écrit F. Cumont et comme en écho au mot célèbre d'E. Renan : «Si le christianisme eût été arrêté dans sa croissance par quelque maladie mortelle, le monde eût été mithriaste ». Cette idée du même Renan qu'« un dieu oriental surtout balança un moment la fortune du christianisme » et qu'il résista « plus que les autres cultes » aux progrès de la religion nouvelle se déchiffrait déjà en 1874 chez G. Boissier, selon qui le culte de Mithra "l'emporte sur tous les autres » aux $\mathrm{IIr}^{\mathrm{e}}$ et $\mathrm{IV}^{\mathrm{e}}$ siècles de notre ère. La même conviction s'affirme en $1886 \mathrm{chez}$ J. Réville qui approuve Renan sans la moindre réserve. On peut se demander si elle n'a pas suggéré à $F$. Cumont le projet de son maître-livre; car elle y reste fortement présente, sinon fondamentale, en même temps que la question de savoir pourquoi le christianisme a triomphé. Renan avait ouvert le débat. C'est Cumont qui devait lui donner toute sa dimension scientifique, en l'étayant sur un appareil textuel et archéologique sans précédent.

Mais il importait, corrélativement, d'en traiter dans le cadre des religions dites « orientales » que G. Boissier appelait simplement « étrangères », conformément à la tradition romaine. Ainsi s'esquissaient les conférences que F. Cumont donnera au Collège de France en 1905 et qu'il publiera dix ans après le tome II des Textes et monuments. Mais tout comme l'idée d'une prééminence mithriaque dans la résistance au christianisme, l'esquisse en était déjà programmée, si j'ose dire, dans le Marc-Aurèle d'E. Renan.

Au chapitre XXXI de ce livre qui, en 1881, concluait une série inaugurée dix-huit ans plus tôt par la Vie de Jésus et que cite à l'occasion F. Cumont, E. Renan veut élucider les Raisons de la victoire du christianisme. Comme toujours s'enchaînent dans son exposé d'élégantes spirales où l'érudition et la poésie, le rêve et l'histoire composent une espèce de symphonie propre à séduire l'imagination du lecteur. Mais, après avoir montré les insuffisances du polythéisme traditionnel ou officiel, la faillite populaire de la philosophie, E. Renan met en scène les religions des « dieux orientaux » : d'abord « les hideux tauroboles » (dont F. Cumont parlera comme d'un « hideux sacrifice »); puis - après un détour par les confréries ou collèges - l'isiasme et ses offices quotidiens qui préfigurent quelque chose du culte chrétien; ensuite les dieux syriens; enfin Mithra et tout ce qui pouvait apparenter sa liturgie à celle des premières «églises». C'est l'ordre même dans lequel F. Cumont nous explique ses Religions orientales dans le paganisme romain.

Certes, cet ordre avait certaines raisons historiques et chronologiques. Le culte de Cybèle est le premier que les Romains adoptèrent officiellement, avant de céder officieusement aux charmes d'Isis. La Déesse Syrienne avait pourtant exalté la fureur sacrée des masses serviles dès le II $^{\mathrm{e}}$ siècle avant J.-C. Mais on ne peut pas dire que Jupiter Dolichenus ait précédé Mithra en Occident. De toute façon, cet enchaînement et ce regroupement même avaient de quoi influencer le jeune F. Cumont. Déjà Firmicus Maternus avait, au nom d'une ethno-théologie des quatre éléments, solidarisé les quatre 
grandes catégories de religions orientales, mais dans un ordre différent (Égypte, Phrygie, Syro-Phénicie, Perse) qu'on retrouve en 1886 chez J. Réville, dans sa Religion à Rome sous les Sévères. L'ordre que suivra F. Cumont est celui d'E. Renan, qui aura marqué aussi bien, je crois, l'idée directrice des Textes et monuments qu'à plus long terme les Religions orientales dans le paganisme romain.

Pourtant - sauf le souci de vérifier la concurrence finale de Mithra et du Christ - cette marque renanienne reste plutôt mineure au total ou, du moins, sans commune mesure avec les chantiers que le savant belge a ouverts, approfondis, élucidés dans l'histoire du paganisme romain, non plus d'ailleurs qu'avec les exigences et la loyauté de sa méthode.

Lorsque F. Cumont entreprit son enquête sur le culte de Mithra, « un archéologue savant et expérimenté chercha (nous dit R. Dussaud) à le dissuader de s'attacher à une étude aussi ingrate ». Preuve qu'il ne faut pas toujours écouter les archéologues, même "savants et expérimentés ", plutôt que la musique d'un historien subtil, à la fois scientiste et dilettante. Mais à condition de savoir outrepasser, comme le pius Aeneas, l'écueil des Sirènes, sans renoncer pour autant aux vertus de l'art et du style.

Quand je parle du style, je ne songe évidemment pas à cet art d'habiller le vague ou le vide qu'on impute indûment au seul académisme, mais au souci de penser assez fortement les choses pour les faire vivre et sentir par les mots. Le vrai style est toujours l'adéquation de la forme au fond. Et cela ne vaut pas seulement pour la science qui, selon Taine (encore un scientiste qui avait du style), s'achève par l'art « comme une plante par sa fleur». Les phénomènes d'ordre spirituel requièrent assurément la science, mais corrélativement aussi la sympathie intime et intuitive, le sens des impondérables et donc une écriture sans caricature, capable de suggérer une part des angoisses ou des ardeurs aujourd'hui disparues, pour faire participer le lecteur à ces « étranges révolutions morales » qu'E. Renan aimait et excellait à évoquer.

F. Cumont n'y a pas moins excellé, mais dans une forme épousant la solidité du fond. Avec le soubassement de ce tome II désormais séculaire, il évitait les « rochers des Sirènes » pour bâtir, si je puis dire, sur le roc du Pétrogène. Un roc certes inébranlable, mais d'où $F$. Cumont fait surgir un Mithra mazdéen, dont l'hellénisation et la «mystérisation » (pour user d'un néologisme plus commode que le problème à résoudre) continuent d'exciter nos interrogations. Car, finalement, les vrais fondateurs de nos études sont ceux qui suscitent et vivifient la recherche, bien loin d'en pétrifier les bornes ou les buts.

3, Résidence du Tourillon

Robert TurCan

F - 69290 CRAPONNE 